IJMMS 30:12 (2002) 709-715

PII. S0161171202107058

http://ijmms.hindawi.com

(c) Hindawi Publishing Corp.

\title{
HARMONICITY OF HORIZONTALLY CONFORMAL MAPS AND SPECTRUM OF THE LAPLACIAN
}

\author{
GABJIN YUN
}

Received 18 July 2001 and in revised form 29 December 2001

\begin{abstract}
We discuss the harmonicity of horizontally conformal maps and their relations with the spectrum of the Laplacian. We prove that if $\phi: M \rightarrow N$ is a horizontally conformal map such that the tension field is divergence free, then $\phi$ is harmonic. Furthermore, if $N$ is noncompact, then $\phi$ must be constant. Also we show that the projection of a warped product manifold onto the first component is harmonic if and only if the warping function is constant. Finally, we describe a characterization for a horizontally conformal map with a constant dilation preserving an eigenfunction.
\end{abstract}

2000 Mathematics Subject Classification: 53C43, 58E20, 58C40.

1. Introduction. The properties for horizontally conformal maps are studied by many authors (see $[1,2,5,8]$ and the references therein). Since a horizontally conformal map is a Riemannian submersion if the dilation is constant 1 , the notion of horizontally conformal maps is a generalized concept of Riemannian submersions.

Let $M^{n}$ and $N^{m}$ be two Riemannian manifolds of dimensions $n$ and $m$, respectively, and let $\phi: M \rightarrow N$ be a horizontally conformal map with dilation $\rho$. In [8], Kasue and Washio gave curvature formula for horizontally conformal maps which is a generalization of O'Neill's curvature formula for Riemannian submersions. Also it is well known (see $[1,8]$ ) that if $\phi$ has minimal fibers and $\nabla \rho$ is vertical, then $\phi$ is harmonic.

We describe here some characterizations for the harmonicity of horizontally conformal maps. In particular, we consider the projections of a warped product manifold onto each component manifold. Those are examples of horizontally conformal maps. We show that the projection of a warped product manifold onto the first component is a horizontally conformal map if and only if the warping function is constant.

Finally, we consider the spectrum of the Laplacian and its relations with horizontally conformal maps. In [6], Gilkey and Park studied the spectrum of the Laplacian and Riemannian submersions. They proved that a Riemannian submersion $\phi: M \rightarrow N$ commutes with the Laplacian if and only if $\phi^{*}$ preserves the eigenfunctions of the Laplacian. In [10], the author showed that, for horizontally conformal maps, a similar result hold. If a horizontally conformal map preserves an eigenfunction, then the dilation of the horizontally conformal map is given by the square root of the ratio of eigenvalues or a geometric identity must hold.

Throughout, every manifold is connected and smooth, and a compact manifold is assumed to be compact without boundary otherwise stated. 
2. Harmonicity of horizontally conformal maps. In this section, we describe basic notions and properties for horizontally weakly conformal maps, harmonic maps and harmonic morphisms, and their relations.

Let $\left(M^{n}, g\right)$ and $\left(N^{m}, h\right)$ be Riemannian manifolds of dimensions $n$ and $m$, respectively. Let $\phi: M \rightarrow N$ be a smooth map. We say that $\phi$ is a harmonic map if it is a critical point of energy functional, or equivalently the tension field of $\phi$ defined by $\tau(\phi)=\operatorname{trace}(\nabla d \phi)$, which is a section of induced bundle $\phi^{-1} T N$, vanishes. For more detail for harmonic maps, see [4]. The covariant derivative of $d \phi, \nabla d \phi$ is called the second fundamental form of $\phi$.

Now for each point $x \in M$, the vertical space of $\phi$ at $x$ is defined by $T_{x}^{V} M=$ $\operatorname{ker}\left(d \phi_{x}\right)$. Let $T_{x}^{H} M$ denote the orthogonal complement of $T_{x}^{V} M$ in the tangent space $T_{x} M$, called the horizontal space. Let $T^{V} M$ and $T^{H} M$, respectively, denote the corresponding vertical and horizontal distributions in the tangent bundle $T M$. We say that $\phi$ is horizontally (weakly) conformal if, for each point $x \in M$ at which $d \phi_{x} \neq 0$, the restriction $\left.d \phi_{x}\right|_{T^{H} M}: T_{x}^{H} M \rightarrow T_{\phi(x)} N$ is conformal and surjective. Thus, in this case, there is a nonnegative function $\rho$ on $M$ satisfying

$$
\phi^{*} h=\rho^{2} g \quad \text { on } T^{H} M .
$$

The function $\rho$ is called the dilation of $\phi$. Note that $\rho^{2}$ is a smooth function and actually equal to $|d \phi|^{2} / m$, where $m=\operatorname{dim}(N)$.

On the other hand, harmonic morphisms are maps preserving the harmonic structures of Riemannian manifolds. More precisely, $\phi$ is called a harmonic morphism if for any function $f$ which is harmonic on an open subset $U$ of $N$ with $\phi^{-1}(U) \neq \varnothing$, the composition $f \circ \phi$ is harmonic on $\phi^{-1}(U)$. Fuglede [5] and Ishihara [7] proved that a smooth map $\phi: M \rightarrow N$ is a harmonic morphism if and only if $\phi$ is both harmonic and horizontally conformal.

Before going on, we would like to mention a formula for the tension field of a horizontally conformal map (see $[1,8]$ ). Let $\phi: M \rightarrow N$ be a horizontally conformal map. Then, the tension field of $\phi$ is given by

$$
\tau(\phi)=d \phi\left(\left(1-\frac{m}{2}\right) \nabla \log \rho^{2}-\kappa\right)
$$

on $M_{0}=\left\{x \in M: d \phi_{x} \neq 0\right\}$, where $n=\operatorname{dim}(M), m=\operatorname{dim}(N)$, and $\kappa$ is the mean curvature of the fibers.

Here we prove the characterization theorem due to Fuglede [5] in case $\operatorname{dim}(M)=$ $\operatorname{dim}(N)$.

THEOREM 2.1 (see [5]). Let $\phi:(M, g) \rightarrow(N, h)$ be a horizontally conformal map with dilation $\rho$. Assume that $\operatorname{dim}(M)=\operatorname{dim}(N)$. Then $\rho$ is constant if and only if $\phi$ is harmonic and so harmonic morphism.

Proof. Let $\operatorname{dim}(M)=\operatorname{dim}(N)=n$. Then, the mean curvature of the fibers vanishes trivially and so (2.2) reduces to

$$
\tau(\phi)=(2-n) d \phi(\nabla \log \rho) .
$$

If $n=2$, then from (2.3), we obviously obtain $\tau(\phi)=0$. 
Now assume that $n \geq 3$. Since $\phi$ is a horizontally conformal with dilation $\rho$, taking the norm of tension field $\tau(\phi)$, we have

$$
|\tau(\phi)|=(n-2)|\nabla \rho| .
$$

Hence $\rho$ is constant if and only if $\tau(\phi)=0$, that is, $\phi$ is harmonic.

Now we consider a general case, that is, the case when $\operatorname{dim}(M) \neq \operatorname{dim}(N)$. In [9], Ishihara and Yano proved that if $M$ is compact and $\phi: M \rightarrow N$ is a smooth map, such that the tension field $\tau(\phi)$ is parallel, then $\phi$ is harmonic. For a horizontally conformal map, a similar result holds with a weaker condition.

THEOREM 2.2. Let $M$ be a compact manifold and let $\phi:\left(M^{n}, g\right) \rightarrow\left(N^{m}, h\right)$ be a horizontally conformal map. If $\tau(\phi)$ is divergence free, then $\phi$ is harmonic and so is a harmonic morphism. Furthermore, if $N$ is noncompact, $\phi$ must be constant.

Proof. Assume that $n \geq m$. Recall that (see [9])

$$
\operatorname{div}(d \phi \cdot \tau(\phi))=|\tau(\phi)|^{2}+\langle d \phi, \nabla(\tau(\phi))\rangle .
$$

Choose an orthonormal frame $\left\{e_{1}, \ldots, e_{n}\right\}$ so that $\left\{e_{1}, \ldots, e_{m}\right\}$ is a basis for $T^{H} M$ and $\left\{e_{m+1}, \ldots, e_{n}\right\}$ is a basis for $T^{V} M$. Then $\left\{d \phi\left(e_{i}\right) / \rho\right\}_{i=1}^{m}$ becomes a local orthonormal frame on $N$ at which $\rho \neq 0$. Thus, it is easy to see for a horizontally conformal map,

$$
\begin{aligned}
\langle d \phi, \nabla(\tau(\phi))\rangle & =\sum_{i=1}^{m}\left\langle d \phi\left(e_{i}\right), \nabla_{e_{i}}^{\phi^{-1} T N} \tau(\phi)\right\rangle_{N} \\
& =\sum_{i=1}^{m}\left\langle d \phi\left(e_{i}\right), \nabla_{d \phi\left(e_{i}\right)}^{N} \tau(\phi)\right\rangle_{N} \\
& =\rho^{2} \operatorname{div}(\tau(\phi)) \circ \phi=0 .
\end{aligned}
$$

Integrating (2.5) over $M$, we have $\tau(\phi)=0$ by Stokes' theorem. Hence $\phi$ is harmonic and so a harmonic morphism.

On the other hand, it is well known (see [5]) that a harmonic morphism is an open map and so $\phi(M)$ is open in $N$. Also since $\phi(M)$ is compact, it is closed. Consequently, if $\phi$ is nonconstant, $\phi(M)=N$ since both $M$ and $N$ are connected.

If $\phi:\left(M^{n}, g\right) \rightarrow\left(N^{m}, h\right)$ is a harmonic morphism with constant dilation $\rho$ and $f$ is a nonconstant eigenfunction $f$ on $N$, that is, $\Delta_{N} f=-\lambda f$ for some $\lambda \in \mathbb{R}$, then the composition $f \circ \phi$ is an eigenfunction on $M$. In fact, it is easy to compute the Hessian of the composition $f \circ \phi$,

$$
\nabla d(f \circ \phi)=d f \circ \nabla d \phi+\nabla d f(d \phi, d \phi) .
$$

Let $\phi$ be a horizontally conformal map with dilation $\rho$. Choose a local orthonormal frame $\left\{e_{1}, \ldots, e_{n}\right\}$ on $M$ so that $\left\{e_{1}, \ldots, e_{m}\right\}$ is a basis for the horizontal space $T^{H} M$ and $\left\{e_{m+1}, \ldots, e_{n}\right\}$ is a basis for the vertical space $T^{V} M$. Then $\left\{d \phi\left(e_{i}\right) / \rho\right\}_{i=1}^{m}$ is a local orthonormal frame on $N$ at which $\rho \neq 0$. Taking the trace of $\nabla d(f \circ \phi)$ with respect to $\left\{e_{i}\right\}$ in (2.7), we obtain

$$
\Delta_{M}(f \circ \phi)=(d f \circ \phi) \cdot \tau(\phi)+\rho^{2}\left(\Delta_{N} f\right) \circ \phi,
$$


and so harmonicity of $\phi$ and the hypothesis that $f$ is an eigenfunction imply

$$
\Delta_{M}(f \circ \phi)=-\lambda \rho^{2}(f \circ \phi) .
$$

For harmonicity of a horizontally conformal map related with eigenfunctions, we have the following theorem.

THEOREM 2.3. Let $\phi:\left(M^{n}, g\right) \rightarrow\left(N^{m}, h\right)$ be a horizontally conformal map with constant dilation $\rho$. Let $f$ be a nonconstant eigenfunction $f$ on $N$, that is, $\Delta_{N} f=-\lambda f$ for some $\lambda \in \mathbb{R}$. If $\nabla(f \circ \phi)$ is vertical, then $\phi$ is harmonic and so is a harmonic morphism.

Proof. Recall that from (2.2) and the assumption that $\rho$ is constant,

$$
\tau(\phi)=-d \phi(\kappa)
$$

Then,

$$
(d f \circ \phi) \cdot \tau(\phi)=-(d f \circ \phi) \cdot d \phi(\kappa)=-\langle\nabla(f \circ \phi), \kappa\rangle=0 .
$$

The last equality follows from the facts that $\nabla(f \circ \phi)$ is vertical and the mean curvature, $\kappa$, of the fibers is horizontal. Since $f$ is nonconstant, the set $\left\{y \in N: d f_{y}=0\right\}$ is discrete in $N$ and so $\tau(\phi)=0$ on $M$.

Next, we consider the Riemannian submersions related with warped product manifolds. Riemannian submersions are special cases of horizontally conformal maps. In fact, a Riemannian submersion is a horizontally conformal map with dilation $\rho=1$.

Let $\left(B, g_{B}\right)$ and $\left(F, g_{F}\right)$ be Riemannian manifolds and let $\alpha$ be a positive smooth function defined on $B$. Then, a product manifold $M=B \times_{\alpha} F$ with metric $g=g_{B}+\alpha^{2} g_{F}$ is called the warped product manifold.

THEOREM 2.4. Let $\phi: M=B \times{ }_{\alpha} F \rightarrow B$ be the projection defined by $\phi(x, y)=x$. Then $\phi$ is a harmonic map if and only if $\alpha$ is constant.

Proof. Note that $\phi$ is a Riemannian submersion. Thus, $\phi$ is harmonic if and only if the fibers are minimal submanifolds of $M$.

Let $x \in B$ be a point and consider the fiber $\phi^{-1}(x)=F$ and the inclusion

$$
\iota:\left(\phi^{-1}(x), f(x)^{2} g_{F}\right) \longrightarrow(M, g) .
$$

Define a tensor (normal connection) $T$ on $\phi^{-1}(x)$ by

$$
T_{V} W=\left(\nabla_{V} W\right)^{\perp}=\nabla d \iota(V, W) \in T^{H} M .
$$

Then the mean curvature vector, $\kappa$, is defined by $\kappa=\operatorname{trace}(T)$. Recall (see [3]) that for warped product metric $g$,

$$
T_{V} W=-\langle V, W\rangle_{g} \frac{\nabla \alpha}{\alpha} .
$$

Choosing an orthonormal basis $\left\{E_{1}, \ldots, E_{p}\right\}$ on $\phi^{-1}(x)$, obtain

$$
\kappa=\sum_{i=1}^{p} T_{E_{i}} E_{i}=-p \frac{\nabla \alpha}{\alpha},
$$


where $p=\operatorname{dim}(F)$. Therefore, $\phi^{-1}(x)$ is minimal if and only if $\nabla \alpha(x)=0$. Hence, $\phi$ is harmonic if and only if for any $x \in B$, the fiber $\phi^{-1}(x)$ is minimal, or equivalently $\nabla \alpha=0$.

EXAMPLE 2.5. Let $M=(0, \infty) \times_{r} S^{n-1}$ with flat metric $g=d r^{2}+r^{2} g_{0}$, where $g_{0}$ is the standard metric on $S^{n-1}$. Then, the projection $\phi: M \rightarrow(0, \infty), \phi(r, x)=r$ is obviously a Riemannian submersion. However, $\phi$ is not a harmonic map since $\alpha(r)=r$ is not constant. In fact, if $\phi$ is harmonic, then $\phi$ is a harmonic function. But the Laplacian for $g$ is given by

$$
\Delta=\frac{\partial^{2}}{\partial r^{2}}+\frac{(n-1)}{r} \frac{\partial}{\partial r}+\Delta_{S^{n-1}}
$$

So $\Delta \phi=0$ if and only if $\alpha^{\prime}=0$.

EXAMPLE 2.6. Let $M=B \times_{\alpha} F$ be a warped product manifold. Define $\psi: M \rightarrow F$ by $\psi(x, y)=y$. Then, $\psi$ is a harmonic morphism with dilation $\alpha$ and the fibers $\psi^{-1}(y)$ are totally geodesic submanifolds of $M$ and so are minimal.

3. Spectrum and horizontally conformal maps. In this section, we discuss the spectrum of the Laplacian and its relations with horizontally conformal maps. In [6], Gilkey and Park studied the spectrum of the Laplacian and Riemannian submersions. They showed that a Riemannian submersion $\phi: M \rightarrow N$ satisfies $\Delta_{M} \phi^{*}=\phi^{*} \Delta_{N}$ for functions on $N$ if and only if $\phi^{*}$ preserves the eigenfunctions of the Laplacian $\Delta_{N}$. We say that $\phi^{*}: C^{\infty}(N) \rightarrow C^{\infty}(M)$ preserves the eigenfunctions of the Laplacian if for any $\lambda \in \mathbb{R}$ there exists $\mu=\mu(\lambda) \in \mathbb{R}$ such that

$$
\phi^{*} E\left(\lambda, \Delta_{N}\right) \subset E\left(\mu, \Delta_{M}\right),
$$

where $E\left(\lambda, \Delta_{N}\right)$ is the eigenvalue defined by

$$
E\left(\lambda, \Delta_{N}\right)=\left\{f \in C^{\infty}(N): \Delta_{N} f=-\lambda f\right\},
$$

and $E\left(\mu, \Delta_{M}\right)$ is defined similarly. In [10], the author proved similar results for horizontally conformal maps. That is, a horizontally conformal map $\phi: M \rightarrow N$ between Riemannian manifolds commutes with the Laplacian if and only if it preserves an eigenvalue. Furthermore if $\phi$ is surjective, the dilation is given by the square of the ratio of the eigenvalues. For a horizontally conformal map which is not surjective, we have the following property.

THEOREM 3.1. Let $\phi:\left(M^{n}, g\right) \rightarrow\left(N^{m}, h\right)$ be a horizontally conformal map with constant dilation $\rho$. Assume that $M$ is compact. Suppose that $f \in E\left(\lambda, \Delta_{N}\right)$ and $f \circ \phi \in$ $E\left(\mu, \Delta_{M}\right)$. Then $\rho=\sqrt{\mu / \lambda}$ or

$$
\int_{M}\langle\nabla(f \circ \phi), \kappa\rangle=0
$$

or equivalently

$$
\int_{M}(f \circ \phi) \operatorname{div}(\kappa)=0
$$

where div denotes the divergence operator. 
Proof. If $\lambda=0$, then $f$ is constant and so $\nabla(f \circ \phi)=0$. We may assume that $\lambda \neq 0$ and $\rho \neq \sqrt{\mu / \lambda}$. Recall that (2.8)

$$
\Delta_{M}(f \circ \phi)=(d f \circ \phi) \cdot \tau(\phi)+\rho^{2}\left(\Delta_{N} f\right) \circ \phi .
$$

Thus

$$
\left(\rho^{2} \lambda-\mu\right) f \circ \phi=d f \circ \tau(\phi)
$$

and so

$$
\left(\rho^{2} \lambda-\mu\right) f \circ \phi=d f \circ \tau(\phi)=\langle\nabla(f \circ \phi), \kappa\rangle .
$$

Since $\rho^{2} \lambda-\mu \neq 0$ and $M$ is compact, we obtain

$$
\int_{M}\langle\nabla(f \circ \phi), \kappa\rangle=0 .
$$

EXAMPLE 3.2. Let $B$ and $F$ be compact Riemannian manifolds and let $M=B \times{ }_{\alpha} F$ be a warped product manifold. Define the projection $\phi: M \rightarrow B$ by $\phi(x, y)=x$. Suppose that $f \in E\left(\lambda, \Delta_{B}\right)$ and $f \circ \phi \in E\left(\mu, \Delta_{M}\right)$. Then $\phi$ is a Riemannian submersion and so it follows from [6] that $\lambda=\mu$.

On the other hand, if $\nabla(f \circ \phi)$ is vertical, then $\phi$ is harmonic and so $\alpha$ is constant. Since $\nabla(f \circ \phi)=\nabla_{B} f, \nabla(f \circ \phi)$ is vertical if and only if $f$ is constant or equivalently $\lambda=0=\mu$.

EXAMPLE 3.3. Let $B$ and $F$ be compact Riemannian manifolds and let $M=B \times{ }_{\alpha} F$ be a warped product manifold. Define the projection $\psi: M \rightarrow F$ by $\psi(x, y)=y$. Suppose that $f \in E\left(\lambda, \Delta_{F}\right)$ and $f \circ \phi \in E\left(\mu, \Delta_{M}\right)$. Then $\psi$ is a harmonic morphism with dilation $\alpha$. If $\lambda$ is not zero, then it follows from [10] that $\alpha$ is constant given by $\sqrt{\mu / \lambda}$. Hence, $M=B \times{ }_{\alpha} F$ is a product manifold up to homothety.

ACKNOWLEDGMENT. This work was supported by grant No. R02-2000-00013 from the Basic Research Program of the Korea Science and Engineering Foundation.

\section{REFERENCES}

[1] P. Baird, Harmonic Maps with Symmetry, Harmonic Morphisms and Deformations of Metrics, Research Notes in Mathematics, vol. 87, Pitman, Massachusetts, 1983.

[2] P. Baird and S. Gudmundsson, $p$-harmonic maps and minimal submanifolds, Math. Ann. 294 (1992), no. 4, 611-624.

[3] R. L. Bishop and B. O'Neill, Manifolds of negative curvature, Trans. Amer. Math. Soc. 145 (1969), 1-49.

[4] J. Eells and L. Lemaire, Selected Topics in Harmonic Maps, CBMS Regional Conference Series in Mathematics, American Mathematical Society, Rhode Island, 1983.

[5] B. Fuglede, Harmonic morphisms between Riemannian manifolds, Ann. Inst. Fourier (Grenoble) 28 (1978), no. 2, 107-144.

[6] P. B. Gilkey and J.-H. Park, Riemannian submersions which preserve the eigenforms of the Laplacian, Illinois J. Math. 40 (1996), no. 2, 194-201.

[7] T. Ishihara, A mapping of Riemannian manifolds which preserves harmonic functions, J. Math. Kyoto Univ. 19 (1979), no. 2, 215-229.

[8] A. Kasue and T. Washio, Growth of equivariant harmonic maps and harmonic morphisms, Osaka J. Math. 27 (1990), no. 4, 899-928. 
[9] K. Yano and S. Ishihara, Harmonic and relatively affine mappings, J. Differential Geometry 10 (1975), no. 4, 501-509.

[10] G. Yun, Horizontally conformal maps and eigenfunctions of the Laplacian, Int. Math. J. 2 (2002), no. 5, 445-451.

Gabjin Yun: Department of Mathematics, Myong Ji University, SAN 38-2, NAMdong, YONGIN, KYUNGGI 449-728, KOREA

E-mail address: gabjin@wh .myongji .ac. kr 


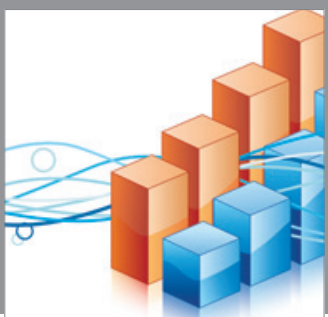

Advances in

Operations Research

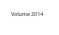

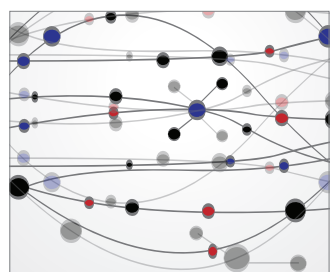

\section{The Scientific} World Journal
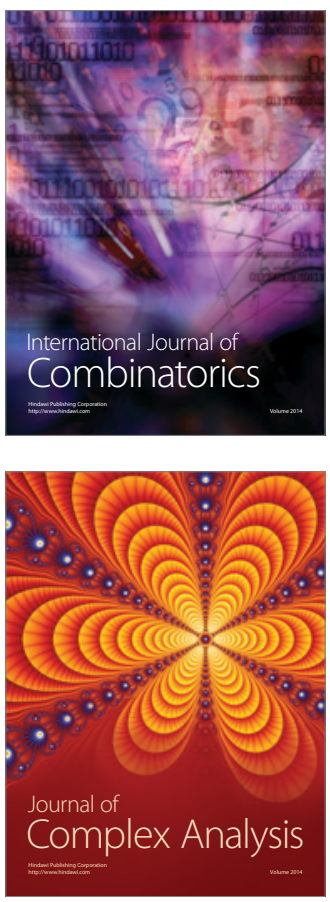

International Journal of

Mathematics and

Mathematical

Sciences
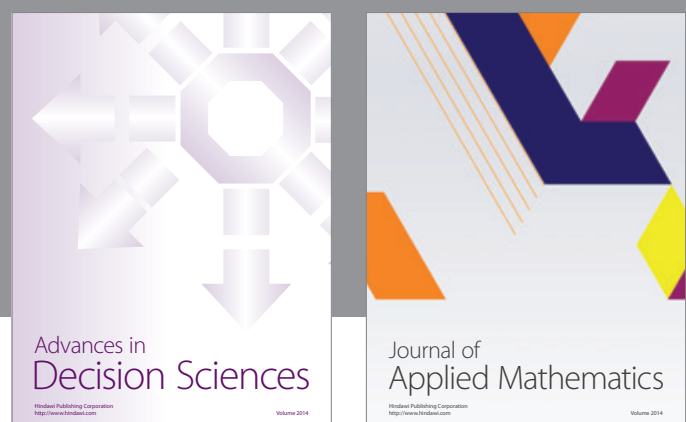

Journal of

Applied Mathematics
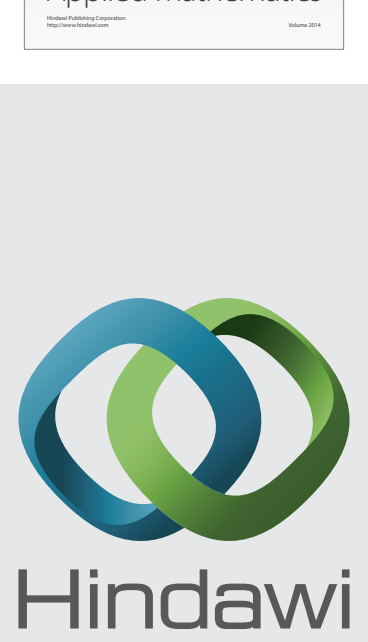

Submit your manuscripts at http://www.hindawi.com
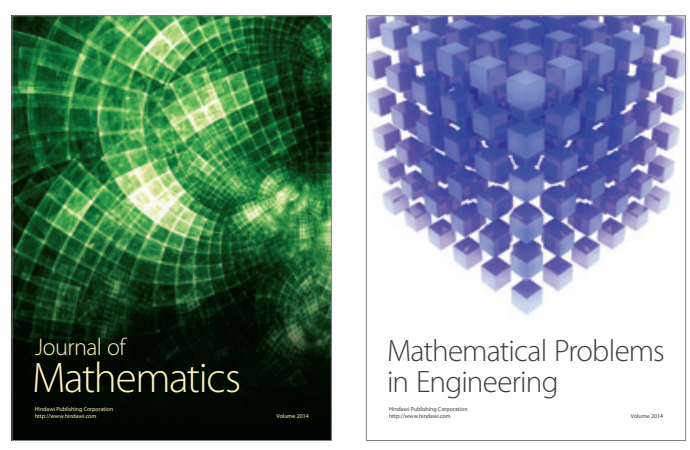

Mathematical Problems in Engineering
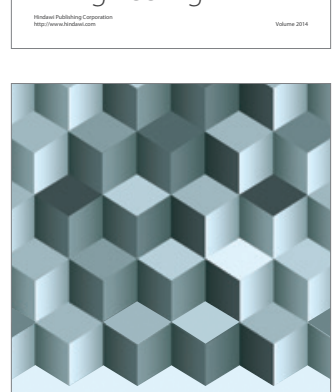

Journal of

Function Spaces
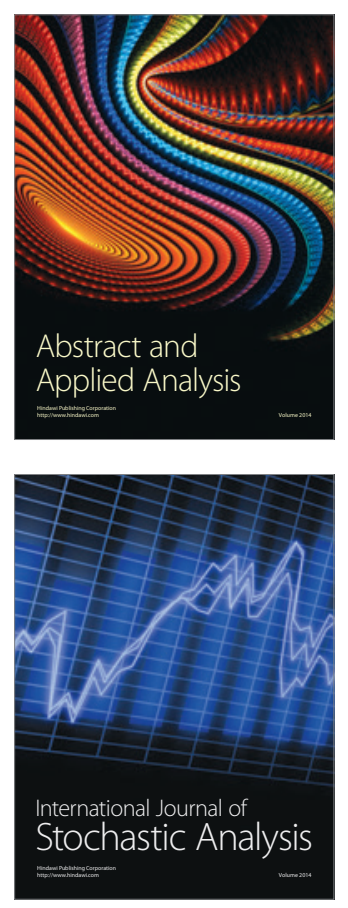

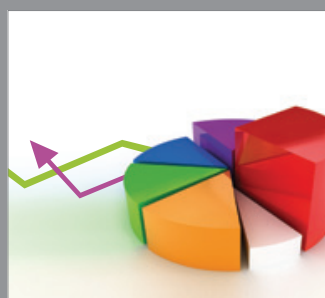

ournal of

Probability and Statistics

Promensencen
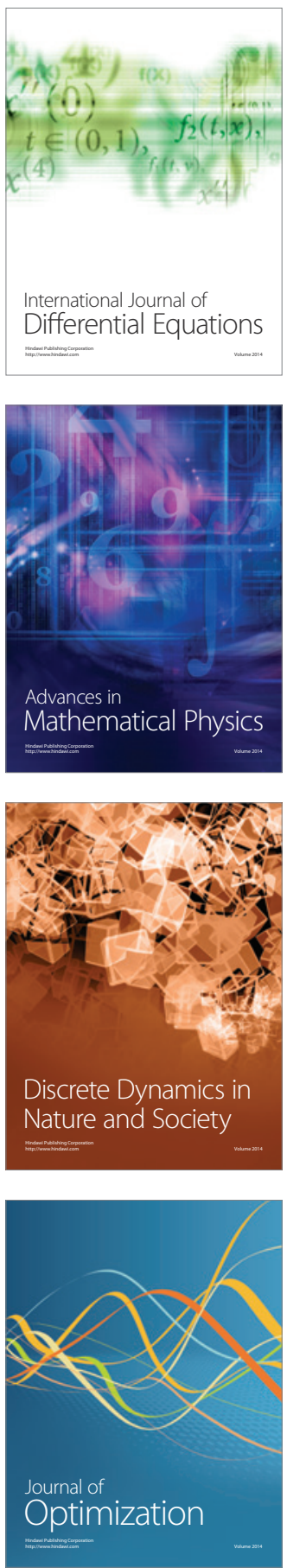